\title{
Promoting Professional Development of Secondary- School Teachers in Greece: An In-School Training in Managing Student Behavior
}

\author{
Sofia Moutiaga ${ }^{1}$, Ioanna Papavassiliou-Alexiou ${ }^{2}$ \\ Department of Educational and Social Policy, University of Macedonia, Thessaloniki, Greece
}

\begin{abstract}
The present paper describes the design and implementation of an in-school teacher training in managing student behavior and investigates its impact on teachers and their students. The training was based on adult learning and group-leading strategies (development-application) as well as Social-Emotional-Learning and School-Wide-Positive-Behavior- Support approaches (content). Research questions were formulated as follows: a. Does the participation in the training (i) lead to the formulation of a misbehavior dealing methodology? (ii) affect the use and sense of usefulness of targeted strategies by teachers? (iii) affect the school climate, teachers' self-efficacy, students' behavior and academic performance? and $b$. how consistent are these effects in the long term? The training consisted of training meetings, coaching and distance education and was implemented in a Thessaloniki's middle-school, using a neighbor middleschool as control-school. Impacts' stability was checked by follow-up tests after four and twelve months accordingly. A convergent parallel mixed-methods design was used for data-analysis. Through the training a school's Community of Practice was developed in the intervention school, that defined and supported a positive behavior management methodology and corresponding strategies. Directly after the training completion, teachers-trainees reported increased professional self-efficacy, teachers and students evaluated their school climate as more positive and students' ODRs decreased, compared to control school. Four months later and without the trainer's support, school's Community of Practice gradually weakened but training beneficial effects remain stable. Benefits decreased after one year, reaching pre-training level. Ways to maintain beneficial results and a sustainable in-service teacher's professional development policy are discussed.
\end{abstract}

Keywords: professional learning; tailored training; teacher competencies; teacher-education policy; training-needs assessment. 